\title{
Stepping-stone model with circular Brownian migration
}

\author{
Xiaowen Zhou \\ Department of Mathematica and Statistics, Concordia University \\ Montreal, Canada
}

\begin{abstract}
In this paper we consider a stepping-stone model on a circle with circular Brownian migration. We first point out a connection between Arratia flow and the marginal distribution of this model. We then give a new representation for the stepping-stone model using Arratia flow and circular coalescing Brownian motion. Such a representation enables us to carry out some explicit computation. In particular, we find the Laplace transform for the time when there is only a single type left across the circle.

Keywords: stepping-stone model, circular coalescing Brownian motion, Arratia flow, duality, entrance law
\end{abstract}

2000 Mathematics Subject Classification: Primary 60G57, Secondary 60J65

\section{INTRODUCTION}

Stepping-stone model is a mathematical model for population genetics. A discretesite stepping-stone model describes the simultaneous evolution of interacting populations over a collection of finite or countable colonies. There are mutation, selection and resampling within each colony, and there is migration among different colonies. See Kim53] and Shi88] for some early work.

Continuous-site stepping-stone model was first introduced in [Eva97. It is a process takeing values from the space

$$
\Xi:=\left\{\mu: E \rightarrow M_{1}(\mathbb{K})\right\}
$$

where $E$ denotes the continuous site space, $\mathbb{K}$ denotes the type space, and $M_{1}(\mathbb{K})$ denotes the space of all probability measures on $\mathbb{K}$. Intuitively, such a map $\mu$ simultaneously represents the relative frequencies of different types in populations at various sites. More precisely, for $e \in E$ and $B \subset \mathbb{K}, \mu(e)(B)$ represents the "proportion of the population at the site $e$ processing types from the set $B$ ". The "moments" of the continuous-site stepping-stone model are specified using the so called migration processes taking values in $E$.

In this paper we only consider a stepping-stone model with site space $\mathbb{T}$, a circle of circumference 1 , with type space $\mathbb{K}=[0,1]$, and with Brownian migration on $\mathbb{T}$. We 
call it a stepping-stone model with circular Brownian migration (in short, a SSCBM) and write it as $X$ throughout the paper.

The distribution of SSCBM is uniquely determined by a family of coalescing Brownian motions on $\mathbb{T}$. But we have to go through more notations before we could present the explicit formula.

Given a positive integer $n$, let $\mathcal{P}_{n}$ denote the set of partitions of $\mathbb{N}_{n}:=\{1, \ldots, n\}$. That is, an element $\pi$ of $\mathcal{P}_{n}$ is a collection $\pi=\left\{A_{1}, \ldots, A_{h}\right\}$ of disjoint subsets of $\mathbb{N}_{n}$ such that $\bigcup_{i} A_{i}=\mathbb{N}_{n}$. The sets $A_{1}, \ldots A_{h}$ are the blocks of the partition $\pi$. The integer $h$ is called the length of $\pi$ and is denoted by $|\pi|$. Equivalently, we can think of $\mathcal{P}_{n}$ as the set of equivalence relations on $\mathbb{N}_{n}$ and write $i \sim_{\pi} j$ if $i$ and $j$ belong to the same block of $\pi \in \mathcal{P}_{n}$.

Given $\pi \in \mathcal{P}_{n}$, let

$$
\alpha_{i}:=\min A_{i}, 1 \leq i \leq|\pi| .
$$

$\left\{\alpha_{i}\right\}$ is the collection of minimal elements for $\pi$.

By a circular (instantaneously) coalescing Brownian motion we mean a collection of Brownian motions on $\mathbb{T}$ such that any two of them will move together as soon as they first meet. Given a circular coalescing Brownian motion $\left(Z_{1}, \ldots, Z_{n}\right)$ starting at $\mathbf{e}=\left(e_{1}, \ldots, e_{n}\right)$. For $t>0$, let $\pi^{\mathbf{e}}(t)$ be a $\mathcal{P}_{n}$-valued random partition such that $i \sim \pi^{\mathbf{e}}(t) j$ iff $Z_{i}(t)=Z_{j}(t)$. Then $\pi^{\mathbf{e}}(t)$ is the random partition induced by $\left(Z_{i}(t)\right)$. Write

$$
\Gamma^{\mathbf{e}}(t):=\left\{\alpha_{i}(t): 1 \leq i \leq\left|\pi^{\mathbf{e}}(t)\right|\right\}
$$

for the collections of minimal elements for $\pi^{\mathbf{e}}(t)$.

SSCBM is then a $\Xi$-valued Hunt process $X$ with its transition semigroup specified as following. Given $\mu \in \Xi$ and $n>0$, for any $f_{i} \in C(\mathbb{T}), K_{i} \subset \mathbb{K}, i=1, \ldots, n$,

$$
\mathbb{Q}^{\mu}\left[\prod_{i=1}^{n} \int_{\mathbb{T}} d e_{i} f\left(e_{i}\right) X_{t}\left(e_{i}\right)\left(K_{i}\right)\right]=\int_{\mathbb{T}^{n}} d \mathbf{e} \prod_{i=1}^{n} f\left(e_{i}\right) \mathbb{P}\left[\bigotimes_{i \in \Gamma^{\mathbf{e}}(t)} \mu\left(Z_{i}(t)\right)\left(\bigcap_{j \sim_{\pi^{\mathbf{e}}(t)}} K_{j}\right)\right],
$$

where $\mathbb{Q}^{\mu}$ denotes the probability law of $X$ when its initial value is $\mu$. See Theorem 4.1 in $\mathrm{DEF}^{+} 00$ for a result on a general continuous-site stepping-stone model.

In $\left.\mathrm{DEF}^{+} 00\right]$ a particle representation for $X$ was given using the Poisson random measure on $D_{\mathbb{T}}[0, \infty[\times \mathbb{K}$ and a "look down" scheme similar to that in DK96]. It leads to better insight into the model. In the same spirit we are going to propose another representation for $X$ in this paper.

It was shown in [Eva97] that $X$ degenerates, i.e. for any $t>0$, for almost all $e \in \mathbb{R}$, $X_{t}(e)$ becomes a point mass on some $k \in \mathbb{K}$. A stronger version of this clustering behavior was later shown in $\mathrm{DEF}^{+} 00$ for site-space $\mathbb{T}$ and in Zho03 for site-space 
$\mathbb{R}$. In fact, when the site-space is $\mathbb{R}$ there exists a random partition of $\mathbb{R}$ such that $\mathbb{R}$ is divided into disjoint intervals and $X_{t}(e)$ is a point mass on the same $k \in \mathbb{K}$ for almost all $e$ in each interval. This suggests that we can identify $X_{t}$ with a function $f$ on $\mathbb{T}$ such that $f(e)=k$ whenever $X_{t}(e)=\delta_{k}$. In this way we can identify $X$ with a step-function-valued process.

Let $\Xi^{\prime}$ be the space of $\mathbb{K}$-valued right continuous step functions on $\mathbb{T}$ equipped with the topology inherited from $D_{\mathbb{R}}(\mathbb{T})$. For each $\mu \in \Xi$, we are going to construct a $\Xi^{\prime}$-valued process $\left(X_{t}^{\prime}, t>0\right)$ which can be regarded as SSCBM with initial value $\mu$ under the above-mentioned identification. To this end, we first point out an interesting connection between Arratia flow and SSCBM in Section 2. This connection allows us to specify the entrance law of $X^{\prime}$ using the pre-image of Arratia flow. Then we give an explicit construction of $X^{\prime}$ using Arratia flow and circular coalescing Brownian motion, and we will show that $X^{\prime}$ so defined does have the right distribution under the above-mentioned identification. In this sense $X^{\prime}$ provides a nice version for $X$. Such a representation enables us to compute the distribution of the time when there is only a single type of individuals left across $\mathbb{T}$ in Section 3 . It also allows us to obtain a result on the type that survives eventually.

\section{A Representation of Stepping-Stone model With CirCUlar Brownian MIGRATION}

We adopt some conventions for the rest of this paper. We identify $\mathbb{T}$ with interval $[0,1)$. Whenever we write $\left(e_{1}, \ldots, e_{m}\right) \in \mathbb{T}^{m}$ it implies that $e_{1}, \ldots, e_{m}$ have been already arranged in anti-clockwise order around $\mathbb{T}$. Given $u, v \in \mathbb{T}$, write $[u, v[$ for an interval starting at $u$ and ending at $v$ in anti-clockwise order. Write $v-u$ for the length of the interval $\left[u, v\left[\right.\right.$. For $\left\{k_{i}\right\} \subset \mathbb{K}$ and $\left(e_{1}, \ldots, e_{m}\right) \in \mathbb{T}^{m}$, write $\sum_{i=1}^{m} k_{i} 1\left\{\left[e_{i}, e_{i+1}[\}, e_{m+1}:=e_{1}\right.\right.$, for a right continuous step function on $\mathbb{T}$.

Arratia flow was first introduced in Arr79]. Arratia flow on $\mathbb{T}$ describes the evolution of a stochastic system in which there is one Brownian motion starting at each point in $\mathbb{T}$. Two Brownian motions coalesce once they meet. Formally, the Arratia flow can be defined as a collection $\{\phi(s, t, x): 0 \leq s \leq t, x \in \mathbb{T}\}$ of random variables such that

- the random map $(s, t, x) \mapsto \phi(s, t, x)$ is jointly measurable,

- for each $s$ and $x$, the map $t \mapsto \phi(s, t, x), t \geq s$, is continuous,

- for each $s$ and $t$ with $s \leq t$, the map $x \mapsto \phi(s, t, x)$ is non-decreasing and right-continuous,

- for $s \leq t \leq u, \phi(t, u, \cdot) \circ \phi(s, t, \cdot)=\phi(s, u, \cdot)$,

- for $u>0,(s, t, x) \mapsto \phi(s+u, t+u, x)$ has the same distribution as $\phi$, 
- for $\left(x_{1}, \ldots, x_{m}\right) \in \mathbb{T}^{m}$ the process $\left(\phi\left(0, t, x_{1}\right), \ldots, \phi\left(0, t, x_{m}\right)\right)_{t \geq 0}$ has the same distribution as a circular coalescing Brownian motion starting at $\left(x_{1}, \ldots, x_{m}\right)$.

From the continuity of Brownian sample paths we see that, for each $t>0$, there exists a positive integer valued random variable $N(t)$ and two sequences of random variables $\left(V_{i}(t)\right) \in \mathbb{T}^{N(t)}$ and $\left(U_{i}(t)\right) \in \mathbb{T}^{N(t)}$ such that

$$
\phi(0, t, x)=V_{i}(t) \text { for } x \in\left[U_{i}(t), U_{i+1}(t)[\text { and } i=1, \ldots, N(t) .\right.
$$

In fact, we can even show that

$$
\mathbb{P}[|N(t)|]=1+2 \sum_{n=1}^{\infty} \exp \left\{-n^{2} \pi^{2} t\right\}
$$

where $|N(t)|$ denotes the cardinality for $N(t)$. See Corollary 9.3 in $\left[\mathrm{DEF}^{+} 00\right]$.

For any $\mu \in \Xi$, given $N(t),\left(U_{i}(t), i=1, \ldots, N(t)\right)$ and $\left(V_{i}(t), i=1, \ldots, N(t)\right)$, let $\left\{\kappa_{i}, i=1, \ldots, N(t)\right\}$ be a collection of independent $\mathbb{K}$-valued random variables such that $\kappa_{i}$ follows the distribution $\mu\left(V_{i}(t)\right)$. Define

$$
X_{t}^{\prime}(e)=\sum_{i=1}^{N(t)} \kappa_{i} 1\left\{\left[U_{i}(t), U_{i+1}(t)[\}(e), e \in \mathbb{T} .\right.\right.
$$

We first point out that $X^{\prime}(t)$, when identified as

$$
\sum_{i=1}^{N(t)} \delta_{\kappa_{i}} 1\left\{\left[U_{i}(t), U_{i+1}(t)[\},\right.\right.
$$

is indeed a version of $X_{t}$.

Proposition 2.1. For any $t>0$, with the identification (2.2) $X_{t}^{\prime}$ has the same distribution as $X_{t}$ under $\mathbb{Q}^{\mu}$.

Proof. To determine the distribution of $X_{t}^{\prime}$ we only need to specify joint distributions such as

$$
\mathbb{P}\left\{X_{t}^{\prime}\left(e_{1}\right) \in d k_{1}, \ldots, X_{t}^{\prime}\left(e_{n}\right) \in d k_{n}\right\} .
$$

By definition $\left(\phi\left(0, t, e_{1}\right), \ldots, \phi\left(0, t, e_{n}\right)\right)$ is a circular coalescing Brownian motion starting at $\left(e_{1}, \ldots, e_{n}\right)$. Let $\pi^{\mathbf{e}}(t)$ be the induced partition on $\mathbb{N}_{n}$. For any $k_{i} \in \mathbb{K}, i=$ $1, \ldots, n$, given $\left(U_{i}(t)\right)$ and $\left(V_{i}(t)\right)$ as before, observe that $\phi\left(0, t, e_{i}\right)$ and $\phi\left(0, t, e_{j}\right)$ belong to the same interval $\left[U_{r}(t), U_{r+1}(t)\right.$ [ for some $r$ iff $i \sim_{\pi^{\mathbf{e}}(t)} j$. Also notice that $\phi\left(0, t, e_{i}\right)=\phi\left(0, t, e_{j}\right)$ whenever $i \sim_{\pi^{\mathrm{e}}(t)} j$. Then

$$
\mathbb{P}\left\{\bigcap_{i=1}^{n}\left\{X_{t}^{\prime}\left(e_{i}\right) \in d k_{i}\right\}\right\}=\mathbb{P}\left[\prod_{i \in \Gamma \mathbf{e}(t)} 1\left\{\bigcap_{j \sim \pi^{\mathbf{e}}(t)}\left\{k_{j}=k_{i}\right\}\right\} \mu\left(\phi\left(0, t, e_{i}\right)\right)\left(d k_{i}\right)\right]
$$

An inspection of (2.3) reveals that (1.1) holds for $X^{\prime}(t)$ when it is regarded as $\Xi$-valued. So $X_{t}$ and $X_{t}^{\prime}$ have the same distribution. 
By Proposition 2.1 we may and will suppose that $X_{t}, t>0$, is $\Xi^{\prime}$-valued in the rest of the paper.

We can read off some properties for $X_{t}, t>0$, immediately from Proposition [2.1] First, with probability one $X_{t}$ (as a function of $e$ ) can only take finitely many different values from $\mathbb{K}$. Moreover, if $\mu(e)$ is a diffuse measure for almost all $e \in \mathbb{T}$, then with probability one $X_{t}$ takes different values over different intervals on $\mathbb{T}$, i.e. $X(e)=k$ for $e \in\left[e_{1}, e_{2}\right.$ [ whenever $X\left(e_{1}\right)=k=X\left(e_{2}\right)$. Such properties are also discussed in Section 10 of $\mathrm{DEF}^{+} 00$.

Conditioning on $X_{s}=\sum_{i=1}^{m} k_{i} 1\left\{\left[u_{i}, u_{i+1}[\}\right.\right.$, (1.1) shows that, given $t>s, X_{t}$ can only take values from $\left\{k_{i}\right\}$. Moreover, for any $\left\{k_{j}^{\prime}, j=1, \ldots, n\right\} \subset\left\{k_{i}\right\}$ and any $\left(z_{j}\right) \in \mathbb{T}^{n}$, by (1.1) we can further show that

$$
\begin{aligned}
\mathbb{Q} & \left\{\bigcap_{j=1}^{n}\left\{X_{t}\left(z_{j}\right)=k_{j}^{\prime}\right\} \mid X_{s}=\sum_{i=1}^{m} k_{i} 1\left\{\left[u_{i}, u_{i+1}[\}\right\}\right.\right. \\
& =\mathbb{Q}\left\{\bigcap_{j=1}^{n}\left\{X_{s}\left(Z_{j}(t-s)\right)=k_{j}^{\prime}\right\}\right\},
\end{aligned}
$$

where $\left(Z_{j}\right)$ is a circular coalescing Brownian motion starting at $\left(z_{j}\right)$.

To describe the evolution of $X$ over time we need a Lemma on duality between two circular coalescing Brownian motions.

Fix $\mathbf{y}=\left(y_{1}, \ldots, y_{m}\right) \in \mathbb{T}^{m}$ and $\mathbf{z}=\left(z_{1}, \ldots, z_{n}\right) \in \mathbb{T}^{n}$. Let $\left(Y_{1}, \ldots, Y_{m}\right)$ be an $m$-dimensional circular coalescing Brownian motion starting at $\mathbf{y}$. Let $\left(Z_{1}, \ldots, Z_{n}\right)$ be an $n$-dimensional circular coalescing Brownian motion starting at $\mathbf{z}$. Put

$$
I_{i j}(t, \mathbf{z}):=1\left\{Y _ { i } ( t ) \in \left[z_{j}, z_{j+1}[\}\right.\right.
$$

and

$$
I_{i j}^{\leftarrow}(t, \mathbf{y}):=1\left\{y _ { i } \in \left[Z_{j}(t), Z_{j+1}(t)[\}\right.\right.
$$

for $1 \leq i \leq m$ and $1 \leq j \leq n$. Recall that $z_{n+1}:=z_{1}$ and $Z_{n+1}:=Z_{1}$.

Lemma 2.2. The two $(m \times n)$-dimensional arrays $\left(I_{i j}^{\leftarrow}(t, \mathbf{y})\right)$ and $\left(I_{i j} \rightarrow(t, \mathbf{z})\right)$ have the same distribution.

Proof. We can prove Lemma 2.2 in the same way as Theorem 2.1 in Zho, i.e. we first show that the corresponding duality holds for circular coalescing random walks, and then apply time-space scaling to obtain the desired result for circular coalescing Brownian motions. 
By Lemma 2.2 we can easily derive the following side result concerning a dual relationship for Arratia flow. Such a result was pointed out in Arr79 for coalescing Brownian flow on the real line.

Proposition 2.3. When identified as point processes, $\left(U_{i}(t)\right)$ and $\left(V_{i}(t)\right)$ have the same distribution for any fixed $t>0$.

Proof. For any $\left(z_{i}\right) \in \mathbb{T}^{2 n}$, let $\left(Z_{i}\right)$ be a coalescing Brownian motion starting at $\left(z_{i}\right)$. Consider a sequence of circular coalescing Brownian motions $\left\{\left(\phi\left(0, t, x_{i}^{m}\right)\right)_{i=1}^{m}, m=\right.$ $1,2, \ldots\}$ such that the set $\left\{x_{i}^{m}, i=1, \ldots, m\right\}$ of starting locations approaches to a dense set in $\mathbb{T}$ as $m \rightarrow \infty$. Such a sequence provides an "approximation" for the Arratia flow on $\mathbb{T}$. Then by Lemma 2.2

$$
\begin{aligned}
& \mathbb{P}\left\{\bigcap_{i=1}^{m}\left\{\phi\left(0, t, x_{i}^{m}\right) \notin \bigcup_{j=1}^{n}\left(z_{2 j-1}, z_{2 j}\right)\right\}\right\} \\
& =\mathbb{P}\left\{\bigcap_{i=1}^{m}\left\{x_{i}^{m} \notin \bigcup_{j=1}^{n}\left(Z_{2 j-1}(t), Z_{2 j}(t)\right)\right\}\right\} .
\end{aligned}
$$

On the one hand, taking limits on both sides of (2.5) as $m \rightarrow \infty$, we can show that

$$
\mathbb{P}\left\{\left\{V_{i}(t)\right\} \bigcap \bigcup_{j=1}^{n}\left(z_{2 j-1}, z_{2 j}\right)=\emptyset\right\}=\mathbb{P}\left\{\bigcap_{j=1}^{n}\left\{Z_{2 j-1}(t)=Z_{2 j}(t)\right\}\right\} .
$$

On the other hand, $\left\{U_{i}(t)\right\} \cap\left(z_{2 j-1}, z_{2 j}\right)=\emptyset$ iff $\left(z_{2 j-1}, z_{2 j}\right) \subset\left[U_{i}(t), U_{i+1}(t)[\right.$ for some $i$ iff $\phi\left(0, t, z_{2 j-1}\right)=V_{i}(t)=\phi\left(0, t, z_{2 j}\right)$ for some $i$. Consequently, we also have

$$
\mathbb{P}\left\{\left\{U_{i}(t)\right\} \bigcap \bigcup_{j=1}^{n}\left(z_{2 j-1}, z_{2 j}\right)=\emptyset\right\}=\mathbb{P}\left\{\bigcap_{j=1}^{n}\left\{\phi\left(0, t, z_{2 j-1}\right)=\phi\left(0, t, z_{2 j}\right)\right\}\right\} .
$$

Therefore, $\left(U_{i}(t)\right)$ and $\left(V_{i}(t)\right)$ have the same avoidance function. So, the assertion of this Proposition holds (see Theorem 3.3 of [Kal76]).

Let us go back to the stepping-stone model. We first consider a special initial value $\mu$. Given

$$
\nu=\sum_{i=1}^{m} \delta_{k_{i}} 1\left\{\left[u_{i}, u_{i+1}[\} \in \Xi,\right.\right.
$$

write $\mathbf{Y}=\left(Y_{i}\right)$ for an $m$-dimensional circular coalescing Brownian motion starting at $\mathbf{u}:=\left(u_{i}\right)$ and define

$$
X_{t}^{\prime}=\sum_{i=1}^{m} k_{i} 1\left\{\left[Y_{i}(t), Y_{i+1}(t)[\}, t \geq 0,\right.\right.
$$

with the convention that $1\{[y, y[\}:=0$.

Lemma 2.4. $X^{\prime}$ has the same distribution as $X$ under $\mathbb{Q}^{\nu}$. 
Proof. $\left(X_{t}^{\prime}\right)$ is clearly a Markov process from its definition.

Given $\left\{k_{1}^{\prime}, \ldots, k_{n}^{\prime}\right\} \subset\left\{k_{i}, i=1, \ldots, m\right\}$ and $\left(v_{j}\right) \in \mathbb{T}^{n}$, let $\mathbf{Z}=\left(Z_{j}\right)$ be a circular coalescing Brownian motion starting at $\mathbf{v}:=\left(v_{j}\right)$. Set

$$
g(\mathbf{y} ; \mathbf{z}):=\prod_{j=1}^{n} \sum_{i: k_{i}=k_{j}^{\prime}} 1\left\{\left[y_{i-1}, y_{i}[\}\left(z_{j}\right), \mathbf{y}:=\left(y_{i}\right), \mathbf{z}:=\left(z_{j}\right) .\right.\right.
$$

Lemma 2.2 yields that

$$
\begin{aligned}
\mathbb{P}\left\{\bigcap_{j=1}^{n}\left\{X_{t}^{\prime}\left(v_{j}\right)=k_{j}^{\prime}\right\}\right\} & =\mathbb{P}\left\{\bigcap _ { j = 1 } ^ { n } \left\{\sum_{i=1}^{m} k_{i} 1\left\{\left[Y_{i}(t), Y_{i+1}(t)[\}\left(v_{j}\right)=k_{j}^{\prime}\right\}\right\}\right.\right. \\
& =\mathbb{P}[g(\mathbf{Y}(t) ; \mathbf{v})] \\
& =\mathbb{P}[g(\mathbf{u} ; \mathbf{Z}(t))] \\
& =\mathbb{P}\left\{\bigcap _ { j = 1 } ^ { n } \left\{\sum_{i=1}^{m} k_{i} 1\left\{\left[u_{i}, u_{i+1}[\}\left(Z_{j}(t)\right)=k_{j}^{\prime}\right\}\right\}\right.\right. \\
& =\mathbb{P}\left\{\bigcap_{j=1}^{n}\left\{X_{0}^{\prime}\left(Z_{j}(t)\right)=k_{j}^{\prime}\right\}\right\} .
\end{aligned}
$$

It then follows from Proposition 2.1 and (2.4) that $X^{\prime}$ and $X$ have both the same initial value and the same transition semigroup. So, they have the same distribution.

Now we are ready to construct a representation for $X$ with a general initial value $\mu \in \Xi$. Given $\epsilon>0$, as in (2.1) put

$$
X_{\epsilon}^{\prime}=\sum_{i=1}^{N(\epsilon)} \kappa_{i} 1\left\{\left[U_{i}(\epsilon), U_{i+1}(\epsilon)[\} .\right.\right.
$$

Given $N(\epsilon),\left(U_{1}(\epsilon), \ldots, U_{N(\epsilon)}(\epsilon)\right)$ and $\left(\kappa_{1}, \ldots, \kappa_{N(\epsilon)}\right)$, write $\left(Y_{i}\right)$ for an $N(\epsilon)$-dimensional circular coalescing Brownian motion starting at $\left(U_{i}(\epsilon)\right)$. We further define

$$
X_{t}^{\prime}=\sum_{i=1}^{N(\epsilon)} \kappa_{i} 1\left\{\left[Y_{i}(t-\epsilon), Y_{i+1}(t-\epsilon)[\}, t \geq \epsilon,\right.\right.
$$

again, with the convention that $1\{[y, y[\}:=0$. Combining Proposition 2.1] and Lemma 2.4 we can easily obtain the following result.

Theorem 2.5. Given $\mu \in \Xi$ and $\epsilon>0,\left(X_{t}^{\prime}, t \geq \epsilon\right)$ has the same distribution as $\left(X_{t}, t \geq \epsilon\right)$ under $\mathbb{Q}^{\mu}$.

Remark 2.6. The representation (2.7) suggests that SSCBM can also be thought of as a multi-type, nearest-neighbored voter model on $\mathbb{T}$. See Chapter V in Lig85 for discussions on voter model. 
Remark 2.7. A similar representation can be found for a stepping-stone model with Brownian migration on $\mathbb{R}$. We leave the details to the readers.

\section{The First time When there is ONLY A Single type LEFT}

In this section we are going to study properties of $X$ using the representation given in Section 2.

Treating $\left(X_{t}, t>0\right)$ as $\Xi^{\prime}$-valued, put

$$
T:=\inf \left\{t>0: \exists k \in \mathbb{K}, X_{t}(e)=k, \forall e \in \mathbb{T}\right\} .
$$

$T$ is then the first time when a single type of individuals prevail all over $\mathbb{T}$. It is easy to see from the representation (2.7) that

$$
\mathbb{Q}^{\mu}\{T<\infty\}=1,
$$

for all $\mu \in \Xi$. Now we are going to find the exact distribution for $T$.

We start with a preliminary result which is interesting in its own right. Let $\left(Y_{i}\right)$ be an $m$-dimensional circular coalescing Brownian motion starting at $\left(y_{i}\right) \in \mathbb{T}^{m}, m \geq 2$. Let

$$
T_{m}:=\inf \left\{t>0: Y_{1}(t)=\ldots=Y_{m}(t)\right\}
$$

Proposition 3.1. Given any positive integer $m \geq 2$, we have

$$
\mathbb{P}\left[e^{-\lambda T_{m}}\right]=\sum_{i=1}^{m} \frac{\sinh \left(\left(y_{i+1}-y_{i}\right) \sqrt{\lambda}\right)}{\sinh (\sqrt{\lambda})}, \lambda>0 .
$$

Proof. For $i=1, \ldots, m$, write $S_{i}$ for the time when $Y_{i+1}$ first reaches $Y_{i}$ from the clockwise direction. As usual, we define $Y_{m+1}:=Y_{1}$. Since $\left(Y_{i+1}-Y_{i}\right) / \sqrt{2}$ is again a Brownian motion which starts at $\left(y_{i+1}-y_{i}\right) / \sqrt{2}$ and stops whenever it reaches 0 or $1 / \sqrt{2}, S_{i}$ is then the first time that the Brownian motion $\left(Y_{i+1}-Y_{i}\right) / \sqrt{2}$ reaches $1 / \sqrt{2}$ before it reaches 0 . We thus have

$$
\mathbb{P}\left[e^{-\lambda S_{i}}\right]=\frac{\sinh \left(\left(y_{i+1}-y_{i}\right) \sqrt{\lambda}\right)}{\sinh (\sqrt{\lambda})} .
$$

See Exercise II.3.10 in RY91.

Our key observation is that

$$
\mathbb{P}\left\{T_{m}<t\right\}=\bigcup_{i=1}^{m}\left\{S_{i}<t\right\},
$$

and the events on the right hand side of this equation are disjoint. So, (3.1) follows.

Standard argument gives the following result. 
Corollary 3.2. For any positive integer $m \geq 2$, we have

$$
\mathbb{P}\left[T_{m}\right]=\frac{1}{4}-\frac{1}{4} \sum_{i=1}^{m}\left(y_{i+1}-y_{i}\right)^{3} .
$$

Consequently, $\mathbb{P}\left[T_{m}\right]$ attains its maximum $1 / 4-1 / 4 m^{2}$ iff all the initial values $y_{1}, \ldots, y_{m}$ are equally spaced on $\mathbb{T}$.

Remark 3.3. An explicit expression for the distribution of $T_{m}$ can also be found. By Theorem 4.1.1 in Kni81, we have

$$
\mathbb{P}\left\{S_{i} \leq t\right\}=\sqrt{2}\left(y_{i+1}-y_{i}\right)+\frac{2}{\pi} \sum_{n=1}^{\infty} \frac{(-1)^{n}}{n} \sin \left(\sqrt{2} n \pi\left(y_{i+1}-y_{i}\right)\right) \exp \left\{-n^{2} \pi^{2} t\right\} .
$$

Therefore,

$$
\mathbb{P}\left\{T_{m} \leq t\right\}=\sqrt{2}+\frac{2}{\pi} \sum_{i=1}^{m} \sum_{n=1}^{\infty} \frac{(-1)^{n}}{n} \sin \left(\sqrt{2} n \pi\left(y_{i+1}-y_{i}\right)\right) \exp \left\{-n^{2} \pi^{2} t\right\} .
$$

We expect that $\mathbb{P}\left\{T_{m} \leq t\right\}$ also reaches its minimum when $y_{1}, \ldots, y_{m}$ are equally spaced on $\mathbb{T}$. But we do not have a proof yet.

Let $\kappa$ be the type of individuals left after time $T$. Then

$$
\kappa=\lim _{t \rightarrow \infty} X_{t}(e), \forall e \in \mathbb{T} .
$$

Theorem 3.4. Given $\mu \in \Xi$ such that $\mu(x)$ is a diffuse probability measure for almost all $x \in \mathbb{T}$, then the Laplace transform for $T$ has the expression

$$
\mathbb{Q}^{\mu}\left[e^{-\lambda T}\right]=\frac{\sqrt{\lambda}}{\sinh (\sqrt{\lambda})}, \lambda>0 .
$$

Moreover,

$$
\mathbb{Q}^{\mu}\{\kappa \in d k\}=\int_{\mathbb{T}} d e \mu(e)(d k) .
$$

Proof. Given $m$ and $\left(y_{i}\right) \in \mathbb{T}^{m}$, we first observe that, by (3.1),

$$
\lim _{m \rightarrow \infty} \mathbb{P}\left[e^{-\lambda T_{m}}\right]=\lim _{m \rightarrow \infty} \sum_{i=1}^{m} \frac{\left(y_{i+1}-y_{i}\right) \sqrt{\lambda}}{\sinh (\sqrt{\lambda})}=\frac{\sqrt{\lambda}}{\sinh (\sqrt{\lambda})}
$$

as

$$
\max _{1 \leq i \leq m}\left(y_{i+1}-y_{i}\right) \rightarrow 0+.
$$

Recall from the representation (2.7) that, for $\epsilon>0$,

$$
X_{t}^{\prime}=\sum_{i=1}^{N(\epsilon)} \kappa_{i} 1\left\{\left[Z_{i}(t-\epsilon), Z_{i+1}(t-\epsilon)[\}, t \geq \epsilon\right.\right.
$$


where, given $N(\epsilon),\left(Z_{i}\right)$ is a circular coalescing Brownian motion starting at $\left(U_{i}(\epsilon)\right) \in$ $\mathbb{T}^{N(\epsilon)}$. Put

$$
T(\epsilon):=\inf \left\{t \geq 0: Z_{1}(t)=\ldots=Z_{N(\epsilon)}(t)\right\} .
$$

Notice that, given $N(\epsilon), \kappa_{1}, \ldots, \kappa_{N(\epsilon)}$ are all different since $\mu(e)$ is diffuse for almost all $e \in \mathbb{T}$. Then $\epsilon+T(\epsilon)$ is also the first time when $X^{\prime}(e)$ assumes a single value in $\mathbb{K}$ for all $e \in \mathbb{T}$.

Put

$$
\Delta(\epsilon):=\max _{1 \leq i \leq N(\epsilon)}\left(U_{i+1}(\epsilon)-U_{i}(\epsilon)\right)
$$

It is evident from the definition of Arratia flow and the representation (2.7) that $\Delta(\epsilon) \rightarrow 0$ in probability and

$$
\mathbb{P}\{T(\epsilon)>0\} \rightarrow 1 \text { as } \epsilon \rightarrow 0+
$$

In addition, $\mathbb{Q}^{\mu}\{T>0\}=1$. It follows from Theorem 2.5 and (3.6) that

$$
\begin{aligned}
\mathbb{Q}^{\mu}\left[e^{-\lambda T}\right] & =\lim _{\epsilon \rightarrow 0+} \mathbb{Q}^{\mu}\left[e^{-\lambda T} ; T>\epsilon\right] \\
& =\lim _{\epsilon \rightarrow 0+} \mathbb{P}\left[\mathbb{P}\left[e^{-\lambda(\epsilon+T(\epsilon))} ; T(\epsilon)>0 \mid X_{\epsilon}^{\prime}\right]\right] \\
& =\frac{\sqrt{\lambda}}{\sinh (\sqrt{\lambda})} .
\end{aligned}
$$

Finally, by (1.1) we have

$$
\lim _{t \rightarrow \infty} \mathbb{Q}^{\mu}\left\{X_{t}(e) \in d k\right\}=\lim _{t \rightarrow \infty} \mathbb{P}[\mu(Z(t))(d k)],
$$

where $Z$ is a circular Brownian motion starting at $e \in \mathbb{T}$. (3.5) thus follows.

Remark 3.5. Notice that the distribution of $T$ does not depend on $\mu$ as long as $\mu(x)$ is diffuse for almost all $x \in \mathbb{T}$.

Remark 3.6. Let $X$ be a Brownian motion starting at $0<x<1 / \sqrt{2}$. Put

$$
T_{x}:=\inf \left\{t \geq 0: X_{t}=0 \text { or } 1 / \sqrt{2}\right\}
$$

We observe that, for the $\mu$ in Theorem 3.4 .

$$
\begin{aligned}
\mathbb{Q}^{\mu}\left[e^{-\lambda T}\right] & =\lim _{x \rightarrow 0+} \frac{\sinh (x \sqrt{2 \lambda})}{x \sqrt{2} \sinh (\sqrt{\lambda})} \\
& =\lim _{x \rightarrow 0+} \frac{\mathbb{P}\left[e^{-\lambda T_{x}} ; T_{x}=1 / \sqrt{2}\right]}{\mathbb{P}\left\{T_{x}=1 / \sqrt{2}\right\}} \\
& =\lim _{x \rightarrow 0+} \mathbb{P}\left[e^{-\lambda T_{x}} \mid X_{T_{x}}=1 / \sqrt{2}\right] .
\end{aligned}
$$


Using (3.8) and Theorem 4.1.1 in Kni81] we can further find an explicit expression for $\mathbb{Q}^{\mu}\{T \leq t\}$. For $t>0$,

$$
\begin{aligned}
\mathbb{Q}^{\mu}\{T \leq t\} & =\lim _{x \rightarrow 0+} \frac{1}{\sqrt{2} x}\left(\sqrt{2} x+\frac{2}{\pi} \sum_{n=1}^{\infty} \frac{(-1)^{n}}{n} \sin (\sqrt{2} n \pi x) \exp \left\{-n^{2} \pi^{2} t\right\}\right) \\
& =1+2 \sum_{n=1}^{\infty}(-1)^{n} \exp \left\{-n^{2} \pi^{2} t\right\} .
\end{aligned}
$$

Remark 3.7. It is not hard to see from the proof for Theorem 3.4 that the distribution (3.9) coincides with the distribution of the time when the image of Arratia flow on $\mathbb{T}$ first becomes a set of a single element, i.e. the distribution of

$$
\tau:=\inf \{t \geq 0: \phi(0, t, x)=\phi(0, t, y), \forall x, y \in \mathbb{T}\}
$$

Again, for the $\mu$ given in Theorem [3.4 for any $\epsilon>0$, let interval $\left[U_{\epsilon}^{\prime}, U_{\epsilon}^{\prime \prime}\right.$ [ be the unique interval $\left[U_{i}(\epsilon), U_{i+1}(\epsilon)\left[\right.\right.$ in (2.6) such that $\kappa=k_{i}$; i.e. $\left[U_{\epsilon}^{\prime}, U_{\epsilon}^{\prime \prime}[\right.$ is the collection of sites at time $\epsilon$ whose type eventually prevails.

Proposition 3.8. For the $\mu$ given in Theorem 3.4, as $\epsilon \rightarrow 0+$ both $\left(U_{\epsilon}^{\prime}\right)$ and $\left(V_{\epsilon}^{\prime \prime}\right)$ converge in distribution to a uniform distribution on $\mathbb{T}$.

Proof. Clearly $U_{\epsilon}^{\prime \prime}-U_{\epsilon}^{\prime} \rightarrow 0$ in probability. Therefore, we just need to show that for any $[a, b[\subset \mathbb{T}$,

$$
\lim _{\epsilon \rightarrow 0+} \mathbb{P}\left\{U_{\epsilon}^{\prime} \in[a, b[\}=b-a .\right.
$$

To prove (3.10), we first notice that, given $N(\epsilon)$, events $\left\{S_{i}<\infty\right\}, 1 \leq i \leq N(\epsilon)$, are all disjoint, where $S_{i}$ is defined as in the proof for Proposition 3.1 but for a coalescing Brownian motion starting at $\left(U_{i}(\epsilon), U_{i+1}(\epsilon)\right)$. Consequently, by (3.2)

$$
\begin{aligned}
\mathbb{P}\left\{U_{\epsilon}^{\prime} \in[a, b[\}\right. & =\mathbb{P}\left\{\bigcup _ { 1 \leq i \leq N ( \epsilon ) } \left\{U_{i}(\epsilon) \in\left[a, b\left[, S_{i}<\infty\right\}\right\}\right.\right. \\
& =\mathbb{E}\left\{\sum _ { 1 \leq i \leq N ( \epsilon ) } ( U _ { i + 1 } ( \epsilon ) - U _ { i } ( \epsilon ) ) 1 \left\{U_{i}(\epsilon) \in[a, b[\}\}\right.\right. \\
& =\mathbb{E}\left[U_{n}(\epsilon)-U_{m}(\epsilon)\right],
\end{aligned}
$$

where $m:=\min \left\{i: U_{i}(\epsilon) \in\left[a, b[\}\right.\right.$ and $n:=\max \left\{i: U_{i}(\epsilon) \in[a, b[\}\right.$. Therefore, (3.10) follows readily. 
Remark 3.9. If $\mu \in \Xi$ is arbitrary, we can not find the explicit distribution for $T$ under $\mathbb{Q}^{\mu}$. Nevertheless, similar to the proof for Theorem 3.4 we can still show that

$$
\mathbb{Q}^{\mu}\{T \leq t\} \geq 1+2 \sum_{n=1}^{\infty}(-1)^{n} \exp \left\{-n^{2} \pi^{2} t\right\}, t \geq 0 .
$$

\section{REFERENCES}

[Arr79] R. Arratia. Coalescing Brownian motions on the line. PhD thesis, University of Wisconsin, Madison, 1979.

$\left[\mathrm{DEF}^{+} 00\right]$ P. Donnelly, S.N. Evans, K. Fleischmann, T.G. Kurtz, and X. Zhou. Continuumsites stepping-stone models, coalescing exchangeable partitions, and random trees. Ann. Probab., 28:1063-1110, 2000.

[DK96] P. Donnelly and T. G. Kurtz. A countable representation of the fleming-viot measurevalued diffusion. Ann. Probab., 24:698-742, 1996.

[Eva97] S.N. Evans. Coalescing markov labeled partitions and continuous sites genetics model with infinitely many types. Ann. Inst. H. Poincaré Probab., 33:339-358, 1997.

[Kal76] O. Kallenberg. Random Measures. Academic Press, New York, 1976.

[Kim53] M. Kimura. "stepping-stone" models of population. Technical Report 3, Institute of Genetics, Japan, 1953.

[Kni81] F. B. Knight. Essentials of Brownian Motion and Diffusion. Amer. Math. Soc., Providence, RI, 1981.

[Lig85] T.M. Liggett. Interacting Particle Systems. Springer-Verlag, New York, 1985.

[RY91] D. Revuz and M. Yor. Continuous Martingales and Brownian motion. Springer-Verlag, Berlin, 1991.

[Shi88] T. Shiga. Stepping stone models in population genetics and population dynamics. Stochastic Processes Phys. Engng. Math. Appl., 42:345-355, 1988.

[Zho] X. Zhou. A superprocess involving both branching and coalescing. Preprint 2005. Available at arXiv.math.PR/0504222.

[Zho03] X. Zhou. Clustering behavior of a continuous-sites stepping-stone model with brownian migration. Elect. J. Probab., 8:1-15, 2003. 